\title{
Self-organised Aggregation in Swarms of Robots with Informed Robots
}

\author{
Ziya Firat ${ }^{1}$, Eliseo Ferrante ${ }^{2}$, Nicolas Cambier ${ }^{3}$, and Elio Tuci ${ }^{4}$ \\ 1 The Department of Computer Science, Middlesex University London, UK \\ ${ }^{2}$ Lab. of Socioecology \& Social evolution, KU Leuven, Belgium \\ 3 Université de Technologie de Compiégne, Compiégne, France \\ 4 Université de Namur, Namur, Belgium \\ zf069@live.mdx.ac.uk, eliseo.ferrante@bio.kuleuven.be, \\ nicolas.cambier@utc.fr, elio.tuci@gmail.com
}

\begin{abstract}
In this paper, we study a swarm of robots that has to select one aggregation site in an environment in which two sites are available. It is known in the literature that, in presence of asymmetries in the environment, robot swarms are able to perform a collective choice and aggregate in one among two possible sites, for example the largest of the two. We focus on an aggregation scenario where the environment is morphologically symmetric. The two aggregation sites are identical with only one exception: their colour. In addition, in the swarm only a proportion of robots, that we call the informed robots, possess extra information concerning on which specific site the swarm is required to aggregate. The rest of the robots are non-informed, thus they do not possess the above mentioned extra information. In simulation-based experiments we show that, if no robot in the swarm is informed, the swarm is able to break the symmetry and aggregates on one of the two sites at random. However, the introduction of a small proportion of informed robots is enough to break the symmetry: the majority of the swarm aggregates on the site preferred by the informed robot. Additionally, the swarm is also able to completely aggregate on one of the two sites when only half the robots are informed, independently from the swarm size among those we considered. Finally, we analyse how the time dynamics of the aggregation process depend on the proportion of informed robots.
\end{abstract}

Keywords: swarm robotics $\cdot$ self-organisation $\cdot$ aggregation $\cdot$ informed leaders

\section{Introduction}

Swarm robotics is a sub-domain of a larger research area dedicated to the design and control of multi-robot systems [26,4]. Swarm robotics is characterised by the following distinctive elements: i) the use of distributed embodied control, that is, each robot has is own on-board control system in charge of determining the robot's behaviour; ii) local perception, that is, each robot can sense and communicate only within a given range using sensors and actuators mounted 
on its body; iii) the use of indirect communication: given that the robots of a swarm are "anonymous", any single agent can not selectively choose a specific message receiver, but rather communicate implicitly through modification of the environment in which they operate, by emitting sound or by generating other types of signal that are eventually detected by other agents.

Research in swarm robotics generally focuses on the design of individual control mechanisms underpinning a desired collective response, which emerges in a self-organised way from the interactions of system components (i.e., the robots and their environment) [4]. Examples of such collective responses are area coverage [19], chain formation [27], collective decision-making and task partitioning [23, 25, 29], cooperative transport [1], and collective motion [14].

One of the main building blocks in swarm robotics is collective decisionmaking, the ability to make a collective decision without any centralised leadership, but only via local interaction and communication. Several types of collective behaviours can be seen as instances of collective decision making [30,31], including collective motion where robots have to agree on a common direction of motion, and aggregation where robots have to agree on a common location in the environment. In a seminal study illustrated in [10], the authors study collective decision making in the context of collective motion looking at what happens when implicit leaders are introduced. These special individuals, also called informed individuals, have a preferred direction of motion and they bias the collective decision in that direction. The rest of the swarm does not have any preferred direction of motion, nor is able to recognise informed individuals as such. The authors show that the accuracy of the group motion towards the direction known by the informed agents increases asymptotically as the proportion of informed individuals increases. Moreover, the authors show that larger the group, the smaller the proportion of informed individuals needed to guide the group with a given accuracy.

In swarm robotics, the framework of implicit leaders has been studied mainly in the context of collective motion $[8,13,14]$. Inspired by these works, we study the effect of implicit leaders in another collective behaviour strongly linked to collective decision-making: self-organised aggregation [9, 18, 2]. Aggregation processes are extremely common in biological systems [5], resulting in clusters of agents at common areas in the environment. Self-organised aggregation (i.e., an aggregation process not driven by exogenous forces) has been studied in a variety of biological systems $[11,20]$ and also implemented on distributed robotic systems $[16,15,18]$. Indeed, aggregation is often a necessity for many collective systems as it is a prerequisite for other cooperative behaviours [12, 28].

Aggregation has been studied as a best-of- $n$ collective decision-making problem [30] in [17] and in [7]. In both studies, the authors have considered the presence of two circular aggregation sites in the environment, the only two areas where robots can stop, that are indistinguishable to the robots. In [17], the authors considered two cases: in the first one, where the two sites have equal size, under special circumstances the swarm can break the symmetry and aggregate on one of the two sites at random; in the second one, where the two sites have 
different sizes, robots are able to collectively chose the biggest among the two aggregates. In a setting that is similar to the second one in [17], using a different model, the authors of [7] design a swarm able to select the smallest site that can host the entire swarm, rather than the biggest one.

Differently from the aggregation studies mentioned above, and analogously to the studies performed within collective motion [10], in this paper we introduce "informed" robots in the context of self-organised aggregation, and we study how they impact the aggregation dynamics. Informed robots are members of the swarm that have been informed a priori about the aggregation site to stop on, in an environment that offers multiple sites for aggregation. Apart from the preference on the site on which to aggregate, the behaviour of informed robots is controlled by exactly the same mechanisms of non-informed robots. The roles of informed robots is to influence the aggregation dynamics, in a very indirect way, since none of the robots has any means to discriminate informed from non-informed robots. We perform our study with a series of simulation experiments on the simplest possible scenario, represented by a circular arena with two aggregation sites: the desired one coloured in black, and the one to be avoided, coloured in white. The results of our simulations show interesting relationships between swarm size and proportion of informed agents, both on quality and speed of convergence on the desired aggregation site.

The rest of the paper is structured as follows. Section 2 describes the selforganised aggregation method used. In Section 3, we present the experimental setup and how we study the effect of informed robots. Section 4 presents the results of our study. Finally, in section 5, we discuss the significance of our results for the swarm robotics community, and we point to interesting future directions of work.

\section{Methods}

Each robot is controlled by a probabilistic finite state machine (PFSM, see also Figure 1a), similar to the one employed in $[20,2,9,6]$. The robots' controller is made of three states: Random Walk $(\mathcal{R} \mathcal{W})$, Stay $(\mathcal{S})$, and Leave $(\mathcal{L})$. When in state $\mathcal{R W}$, the movement of the robot is characterised by an isotropic random walk, with a fixed step length ( 5 seconds, at $10 \mathrm{~cm} / \mathrm{s}$ ), and turning angles chosen from a wrapped Cauchy probability distribution characterised by the following PDF [21]:

$$
f_{\omega}(\theta, \mu, \rho)=\frac{1}{2 \pi} \frac{1-\rho^{2}}{1+\rho^{2}-2 \rho \cos (\theta-\mu)}, 0<\rho<1,
$$

where $\mu=0$ is the average value of the distribution, and $\rho$ determines the distribution skewness. For $\rho=0$ the distribution becomes uniform and provides no correlation between consecutive movements, while for $\rho=1$ a Dirac distribution is obtained, corresponding to straight-line motion. In this study $\rho=0.5$. Any robot in state $\mathcal{R} \mathcal{W}$ is continuously performing an obstacle avoidance behaviour. 


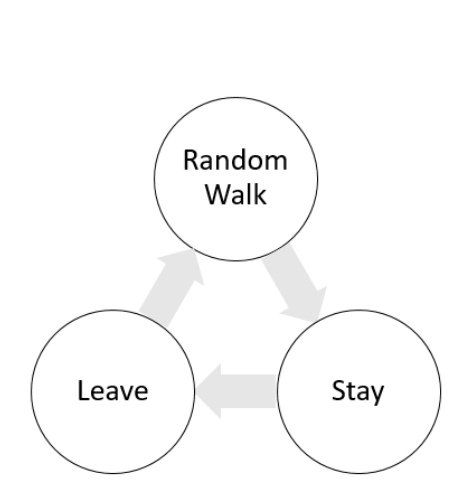

(a)

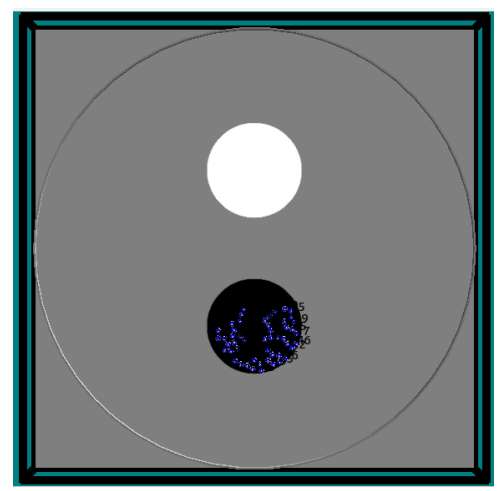

(b)

Fig. 1. (a) State diagram of the robots' controller. (b) The robots' arena with the black and white aggregation site.

To perform obstacle avoidance, first the robot stops, and then it keeps on changing its headings of a randomly chosen angle uniformly drawn in $[0, \pi]$ until no obstacles are perceived.

A robot that, while performing random walk, reaches any of the two aggregation site, it stops with probability $\left(P_{\text {stay }}\right)$. This probability is computed using the following function:

$$
P_{\text {stay }}=0.03+0.48 *\left(1-e^{-a n}\right)
$$

with $n$ corresponding to the number of other robots currently stationing on the site that are perceived by the robot currently deciding whether to stop or not; and $a=0.6$. This function was first introduced in [6]. It interpolates the probability table considered in classical studies such as $[20,9]$. Once the robot has decided to stop based on $P_{\text {stay }}$, it moves forward for a limited number of time in order to avoid stopping at the border of the site thus creating barriers preventing the entrance to other robots, and then transitions from state $\mathcal{R} \mathcal{W}$ to state $\mathcal{S}$. Once in state $\mathcal{S}$ the robot leaves the aggregation site with probability $P_{\text {leave }}$. This probability is computed in the following:

$$
P_{\text {leave }}=e^{-b n}
$$

with $b=2.6$. This function was also introduced in [6]. A robot that decides to leave the aggregation site based on $P_{\text {leave }}$ transitions from state $\mathcal{S}$ to state $\mathcal{L}$. Both $P_{\text {stay }}$ and $P_{\text {leave }}$ are sampled every 20 time steps. When in state $\mathcal{L}$, the robot moves away from the site by moving forward while avoiding collisions with other robots until it no longer perceives the site. At this point, the robot transitions from state $\mathcal{L}$ to state $\mathcal{R} \mathcal{W}$.

In our model we consider two kinds of robots: informed and non-informed. Informed robots are agents that possess extra information on what is the site 
on which the swarm has to aggregate. Ideally, this extra information could be either generated by additional sensors, mounted only on informed robots, which allow these robots to perceive the quality difference between the two aggregation sites, or communicated by the experimenter with the intention to influence the swarm aggregation dynamics. In our simulated scenario, we consider aggregation sites in two different colours: black and white. Informed robots are aware that the task requires to stop only on the black site. Therefore, they ignore the white site, and only stop on the black site based on $P_{\text {stay }}$, as described above. Noninformed robots do not possess this extra information, therefore they can stop both on the black and on the white site based on $P_{\text {stay }}$, as described above.

\section{Experimental Setup}

In this set of simulations, a swarm of robots is randomly initialised in a circular area with the floor coloured in grey except for two circular aggregation sites one coloured in white and one in black (see Figure 1b). The task of the robots is to find and aggregate on the black site. Some of the robots are informed on which site to aggregate. The proportion of informed robots, henceforth denoted as $\rho_{I}$ is systematically varied from $\rho_{I}=0$ (i.e., none of the robots is informed on which site to aggregate) to $\rho_{I}=1$ (all the robots are informed on which site to aggregate) with a step size of 0.1 . We run three different experimental conditions, in which we varied the swarm size. As aggregation performance are heavily influenced by swarm density [6], in this paper we have decided to study scalability by keeping the swarm density constant. Therefore, the diameter of the area, as well as the diameters of the two sites, is varied as well. Table 1 reports a summary of our experimental conditions. In all conditions, the diameter of each aggregation site is large enough to accommodate all the robots of the swarm.

Each experimental condition can be divided in 11 groups which differ in the proportion of informed robots $\rho_{I}$. For each proportion $\rho_{I}$, we execute 200 independent runs. In each run, the robots are randomly initialised within the arena, and then they are left free to act according to actions determined by their PFSM for 10.0000 time steps. One simulated second corresponds to 10 time steps.

We use ARGoS multi engine simulator [24]. The simulation environment models the circular arena as detailed above, and the kinematic and sensors readings of the Foot-bots mobile robots [3]. The robot sensory apparatus includes the proximity sensors positioned around the robot circular body, four ground

Table 1. Table showing the characteristics of each experimental condition.

\begin{tabular}{|c|c|c|c|}
\hline experimental conditions & Swarm size $(\mathrm{N})$ & arena diameter $(\mathrm{m})$ & $\begin{array}{c}\text { aggregation site } \\
\text { diameter }(\mathrm{m})\end{array}$ \\
\hline 1 & 20 & 8.3 & 1.8 \\
\hline 2 & 50 & 12.9 & 2.8 \\
\hline 3 & 100 & 19.2 & 4.0 \\
\hline
\end{tabular}




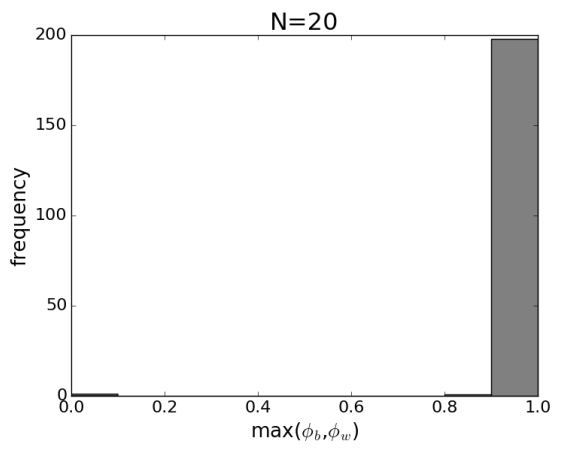

(a)

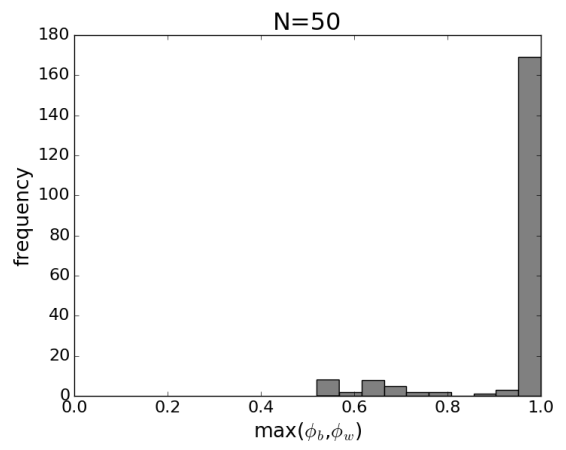

(b)

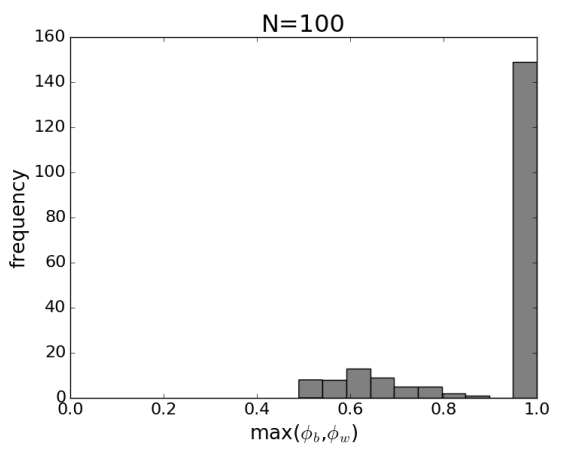

(c)

Fig. 2. Results of the experiments without informed robots $\left(\rho_{I}=0\right)$. The graphs show frequency histograms of the proportion of robots aggregating on the largest aggregate $\left(\max \left(\Phi_{b}, \Phi_{w}\right)\right)$ for swarms of size a) $\mathrm{N}=20$; b) $\mathrm{N}=50$; and c) $\mathrm{N}=100$.

sensors positioned two on the front and two on the back of the robot underside, and the range and bearing sensor. The proximity sensors are used for sensing and avoiding the walls of the arena. The readings of each ground sensors is set to 0.5 if the sensor is on grey, to 1 if on white, and 0 if on black. A robot perceives an aggregation site when all the four ground sensors return a value different from 0.5. Range and bearing sensors are used for inter-robot obstacle avoidance and for sensing the number of neighbours: the robots send a signal whenever they are stationing on a site. These signals are used by the robots to estimate the parameter $n$ necessary to compute $P_{\text {stay }}$ and $P_{\text {leave }}$.

\section{Results}

The main aim of this study is to look at how informed robots influence the aggregation dynamics in a task in which there are two possible aggregation sites, that can be differentiated only by informed robots. To do this, we used 
as performance indicator the proportion of robots aggregated on black site as $\Phi_{b}=\frac{N_{b}}{N}$ and on white site as $\Phi_{w}=\frac{N_{w}}{N}$ (where $N_{b}$ and $N_{w}$ are the number of robots aggregated on the black and white site, respectively) during the last 100.000 time steps of each run. The goal of the swarm is to maximise $\Phi_{b}$ and to minimise $\Phi_{w}$. Note that $\Phi_{b}+\Phi_{w} \leq 1$ as it is possible that not all robots have aggregated in either site by the end of the run.

Prior to testing the effect of informed robots, we conduct a first set of experiments to validate our model. The model we used (see Section 2), is strongly influenced by the work of Garnier et al. [17]. According to this study, in presence of perfectly symmetrical aggregation sites, this aggregation model results in a symmetry breaking, whereby robots tend to chose one of the two sites at random. They aggregate in the chosen site, provided that the site is big enough to host the entire swarm. This symmetry breaking property is an essential feature of a self-organised aggregation method as it provides the positive feedback mechanism necessary for such behaviour. In order to test whether our model has this feature, we have executed experiments without informed robots in order to replicate the results in [17]. To calculate the strength of the positive feedback mechanism, we calculate the proportion of robots aggregated in the largest aggregate as $\max \left(\Phi_{b}, \Phi_{w}\right)$, independently on whether it is on the black or the white site. Results are shown in Figure 2 in form of frequency distribution. The graphs shows that, independently on the swarm size, the distribution looks multi-modal, with the highest peak at 1.0. This indicates that, for all considered swarm sizes, the swarm is able to create large aggregates around one of the sites at random.

We introduce informed robots and analyse how aggregation performance depend on their proportion $\rho_{I}$. The results are shown in Figure 3. We notice that for all swarm size, and when no robot is informed in the swarm $\left(\rho_{I}=0.0\right)$, both $\Phi_{b}$ and $\Phi_{w}$ are centred around 0.5 with a strong variation. As we saw above, this may be explained by the fact that robots chose one aggregate at random under these conditions. Importantly, the introduction of as little as $10 \%$ of informed robots clearly breaks the equal-frequency bimodal aggregation dynamics between the black and the white sites and generates new dynamics that tend to bring the majority (about 60\%) of the robots on the black site. Furthermore, all three graphs show a similar trend in which the higher the number of informed robots, the higher the proportion of robots aggregated on the black site. This trend is non-linear and reaches saturation at around $\rho_{I}=0.5$. With as little as $30 \%$ of informed robots, the majority of the runs finishes with more than $90 \%$ of the robots aggregated on the black site (see black boxes for $\rho_{I}=0.3$ in all graphs in Figure 3). For the smallest swarm size ( $N=20$, see Figure3a) $50 \%$ of informed robots is enough to bring forth very robust and consistent aggregation dynamics that take the entire swarm on the black site. For medium and large swarm, similar robust and consistent dynamics can be observed when the proportion of informed robots is at least $60 \%$. In summary, the above results indicate that with a proportion of informed robots varying from 0.3 to 0.5 of the entire swarm, it is possible to generate robust and consistent aggregation 


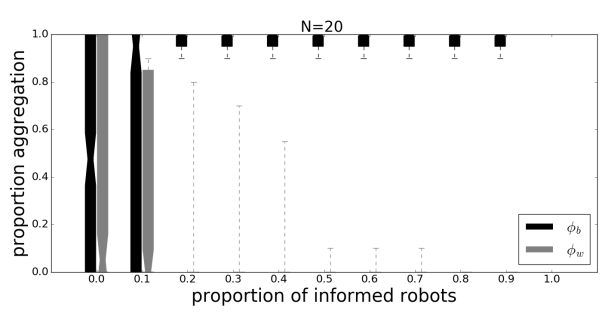

(a)

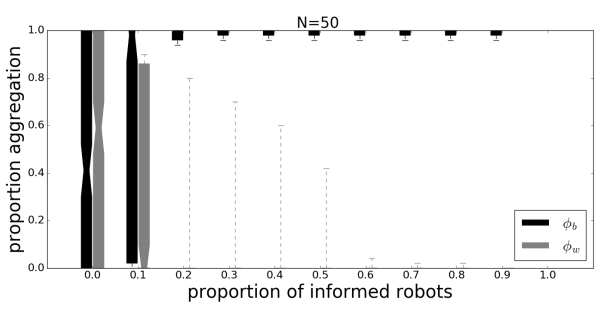

(b)

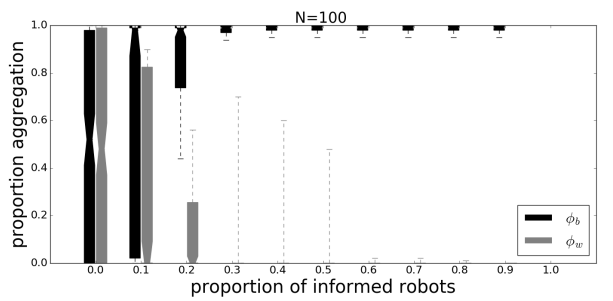

(c)

Fig. 3. Graphs showing the percentage of aggregated robots on the white site (i.e., $\Phi_{w}$, see grey boxes) and on the black site ( $\Phi_{b}$, see black boxes) for swarms of size a) $\mathrm{N}=20$; b) $\mathrm{N}=50$; and c) $\mathrm{N}=100$. In each graph, the $\mathrm{x}$-axis refers to the proportion of informed robots.

dynamics that take the totally of the swarm on a single site, in a task in which two possible aggregation sites are available.

The graphs in Figure 4 show details on the time dynamics of the aggregation process for three different values of $\rho_{I}\left(\rho_{I}=0.1\right.$ in Figure $4 \mathrm{a}, \rho_{I}=0.3$ in Figure $4 \mathrm{~b}$, and $\rho_{I}=0.6$ in Figure $4 \mathrm{c}$ ) and with the largest swarm size $N=100$. All figures feature a non-linear increase of the proportion of robots aggregated on the black site (i.e., $\Phi_{b}$ ), which eventually reaches saturation. By increasing the percentage of informed robots, we initially observe that distribution of convergence values changes dramatically from $\rho_{I}=0.1$ to $\rho_{I}=0.3$. In the latter case, we already observe a good percentage of the runs converging to all robots aggregated on the black site, as the dashed top curve in Figure 4b corresponds to the $75 \%$ percentile. When we increase $\rho_{I}$ to 0.6 , we observe that the variation between the different runs reduces dramatically while converging, and that all quartile of the distributions tend to converge to $\Phi_{b}=1$. Additionally, we can also notice that, the higher the proportion of informed robots, the steeper the slope of the curve during both the first and the second phase. That is, by progressively increasing $\rho_{I}$ the aggregation dynamics unfold in such a way that higher proportion of robots aggregated on the black site appear earlier during the run. To conclude, we can state that both speed (in terms of convergence) and accuracy (in terms of increase of percentage of robots aggregating on the desired site) of the aggregation process increase with increasing proportion of informed robots. 


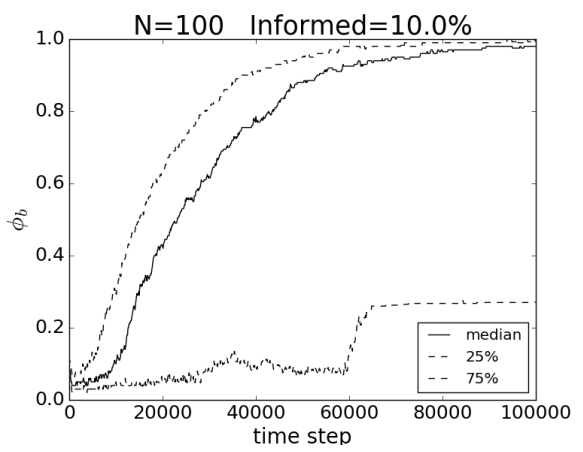

(a)

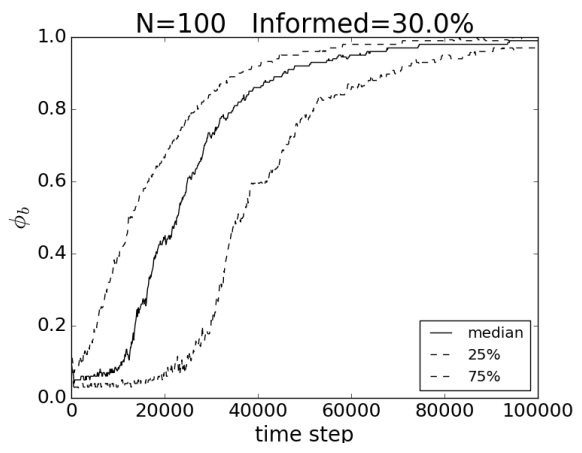

(b)

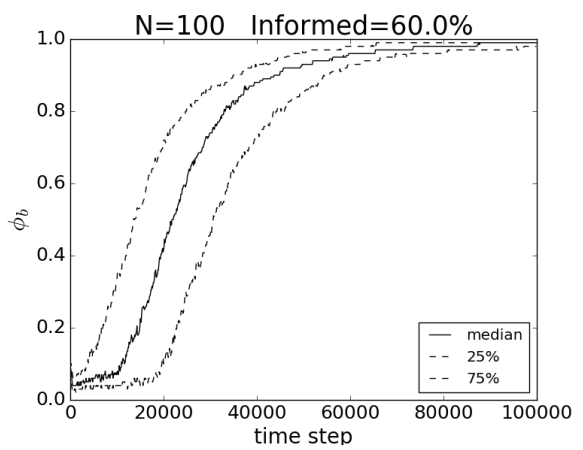

(c)

Fig. 4. Graphs showing the median (see continuous line), the first and third quartile (see dashed lines) of the proportion of robots aggregated on the black site $\left(\Phi_{b}\right)$ at every time step for 200 runs with swarm size $\mathrm{N}=100$. In a) $10 \%$ of the swarm is informed; in b) $30 \%$ of the swarm is informed; in c) $60 \%$ of the swarm is informed.

\section{Conclusions}

In this paper, we have contributed to the wider agenda of studying the role of implicit leaders in the context of collective decision making. We have focused on self-organised aggregation in the simplest possible scenario whereby two symmetrical but differently coloured sites are present. We considered a swarm of robots divided in two sets: informed robots, that possess extra information on which site the swarm has to aggregate, and non-informed robots which do not possess this extra information.

We conducted experiments using the ARGoS simulator in which we varied the proportion of informed robots from $0 \%$ to $100 \%$. Our results show that, in absence of informed robots, robots can either split between the two sites, or break the symmetry randomly by aggregating in one of them. As soon as informed robots are introduced, the symmetry is instead immediately broken 
and increasingly more robots aggregate on the black site. When at least $50 \%$ of the robots are informed, the entire swarm aggregates on the black site, for all swarm sizes we have considered. We have also shown that the speed and accuracy of convergence is also strongly affected by the proportion of informed robots.

This study has the potential to be extended in many possible ways. First, in the context of aggregation, our next step will be to extend the study to more complex scenarios. We plan to test the discrimination capabilities of our swarms with informed robots in environments with more than two aggregation sites. These can include: environments with only one black (i.e., the correct) site and several white sites; environments with multiple black and white sites, in which we require the swarm to aggregate only on one of the black sites; environments with several different options (e.g. colours), which would correspond to a best-of$n$ problem with $n>2$ [30]; scenarios where informed robots may have conflicting information about which is the best site and conflict resolution strategies need to be devised. Secondly, in our vision, we also plan to introduce implicit leaders in other collective behaviours. Finally, our framework can also have a practical relevance in the context of human-swarm interaction [22], whereby informed robots can correspond to robots that are controlled or tele-operated by humans, which would in turn introduce the human in the loop in order to study how humans can interact and control swarm of robots.

\section{References}

1. Alkilabi, M., Narayan, A., Tuci, E.: Cooperative object transport with a swarm of e-puck robots: robustness and scalability of evolved collective strategies. Swarm Intelligence 11(3-4), 185-209 (2017)

2. Bayindir, L., Şahin, E.: Modeling self-organized aggregation in swarm robotic systems. In: IEEE Swarm Intelligence Symposium, SIS'09. pp. 88-95. IEEE (2009)

3. Bonani, M., Longchamp, V., Magnenat, S., Rétornaz, P., Burnier, D., Roulet, G., Vaussard, F., Bleuler, H., Mondada, F.: The marxbot, a miniature mobile robot opening new perspectives for the collective-robotic research. In: IEEE/RSJ Int. Conf. on Intelligent Robots and Systems (IROS). pp. 4187-4193 (2010)

4. Brambilla, M., Ferrante, E., Birattari, M., Dorigo, M.: Swarm robotics: a review from the swarm engineering perspective. Swarm Intelligence 7(1), 1-41 (2013)

5. Camazine, S.: Self-organization in biological systems. Princeton University Press (2003)

6. Cambier, N., Fremont, V., Trianni, V., Ferrante, E.: Embodied evolution of selforganised aggregation by cultural propagation. In: et al., M.D. (ed.) Proc. of the $11^{\text {th }}$ Int. Conf. on Swarm Intelligence. p. In Press. LNCS, Springer (2018)

7. Campo, A., Garnier, S., Dédriche, O., Zekkri, M., Dorigo, M.: Self-organized discrimination of resources. PLoS ONE 6(5), e19888 (2010)

8. Çelikkanat, H., Şahin, E.: Steering self-organized robot flocks through externally guided individuals. Neural Computing and Applications 19(6), 849-865 (Sep 2010)

9. Correll, N., Martinoli, A.: Modeling and designing self-organized aggregation in a swarm of miniature robots (2011)

10. Couzin, I., Krause, J., Franks, N., Levin, S.: Effective leadership and decision making in animal groups on the move. Nature 433, 513-516 (2005) 
11. Deneubourg, J., Lioni, A., Detrain, C.: Dynamics of aggregation and emergence of cooperation. The Biological Bulletin 202(3), 262-267 (2002)

12. Dorigo, M., Trianni, V., Şahin, E., Groß, R., Labella, T., Baldassarre, G., Nolfi, S., Deneubourg, J., Mondada, F., Floreano, D., et al.: Evolving self-organizing behaviors for a swarm-bot. Autonomous Robots 17(2), 223-245 (2004)

13. Ferrante, E., Turgut, A.E., Huepe, C., Stranieri, A., Pinciroli, C., Dorigo, M.: Selforganized flocking with a mobile robot swarm: a novel motion control method. Adaptive Behavior 20(6), 460-477 (2012)

14. Ferrante, E., Turgut, A., Stranieri, A., Pinciroli, C., Birattari, M., Dorigo, M.: A self-adaptive communication strategy for flocking in stationary and non-stationary environments. Natural Computing 13(2), 225-245 (2014)

15. Garnier, S., Jost, C., Gautrais, J., Asadpour, M., Caprari, G., Jeanson, R., Grimal, A., Theraulaz, G.: The embodiment of cockroach aggregation behavior in a group of micro-robots. Artificial life 14(4), 387-408 (2008)

16. Garnier, S., Jost, C., Jeanson, R., Gautrais, J., Asadpour, M., Caprari, G., Theraulaz, G.: Aggregation behaviour as a source of collective decision in a group of cockroach-like-robots. In: European Conference on Artificial Life. pp. 169-178. Springer (2005)

17. Garnier, S., Gautrais, J., Asadpour, M., Jost, C., Theraulaz, G.: Self-organized aggregation triggers collective decision making in a group of cockroach-like robots. Adaptive Behavior 17(2), 109-133 (2009)

18. Gauci, M., Chen, J., Li, W., Dodd, T., Groß, R.: Self-organized aggregation without computation. The International Journal of Robotics Research 33(8), 1145-1161 (2014)

19. Hauert, S., Winkler, L., Zufferey, J., Floreano, D.: Ant-based swarming with positionless micro air vehicles for communication relay. Swarm Intelligence 20 $(2-4)$, 167-188 (2008)

20. Jeanson, R., Rivault, C., Deneubourg, J., Blanco, S., Fournier, R., Jost, C., Theraulaz, G.: Self-organized aggregation in cockroaches. Animal Behaviour 69(1), 169$180(2005)$

21. Kato, S., Jones, M.: An extended family of circular distributions related to wrapped cauchy distributions via brownian motion. Bernoulli 19(1), 154-171 (2013)

22. Kolling, A., Walker, P., Chakraborty, N., Sycara, K., Lewis, M.: Human interaction with robot swarms: A survey. IEEE Transactions on Human-Machine Systems 46(1), 9-26 (Feb 2016). https://doi.org/10.1109/THMS.2015.2480801

23. Montes de Oca, M., Ferrante, E., Scheidler, A., Pinciroli, C., Birattari, M., Dorigo, M.: Majority-rule opinion dynamics with differential latency: a mechanism for selforganized collective decision-making. Swarm Intelligence 5(3-4), 305-327 (2011)

24. Pinciroli, C., Trianni, V., O'Grady, R., Pini, G., Brutschy, A., Brambilla, M., Mathews, N., Ferrante, E., Di Caro, G., Ducatelle, F., Birattari, M., Gambardella, L., Dorigo, M.: ARGoS: a modular, parallel, multi-engine simulator for multi-robot systems. Swarm Intelligence 6(4), 271-295 (2012)

25. Pini, G., Brutschy, A., Frison, M., Roli, A., Dorigo, M., Birattari, M.: Task partitioning in swarms of robots: an adaptive method for strategy selection. Swarm Intelligence 5(3-4), 283-304 (2011)

26. Şahin, E.: Swarm robotics: From sources of inspiration to domains of application. In: International workshop on swarm robotics. pp. 10-20. Springer (2004)

27. Sperati, V., Trianni, V., Nolfi, S.: Self-organised path formation in a swarm of robots. Swarm Intelligence 5(2), 97-119 (2011) 
28. Tuci, E., Alkilabi, M., Akanyety, O.: Cooperative object transport in multi-robot systems: A review of the state-of-the-art. Frontiers in Robotics and AI 5, 1-15 (2018)

29. Tuci, E., Rabérin, A.: On the design of generalist strategies for swarms of simulated robots engaged in a task-allocation scenario. Swarm Intelligence 9(4), 267-290 (2015)

30. Valentini, G., Ferrante, E., Dorigo, M.: The best-of-n problem in robot swarms: Formalization, state of the art, and novel perspectives. Frontiers in Robotics and AI 4, 9 (2017). https://doi.org/10.3389/frobt.2017.00009, https://www.frontiersin.org/article/10.3389/frobt.2017.00009

31. Valentini, G., Ferrante, E., Hamann, H., Dorigo, M.: Collective decision with 100 Kilobots: Speed versus accuracy in binary discrimination problems. Autonomous Agents and Multi-Agent Systems 30(3), 553-580 (2016) 